\title{
Modulation of gene expression dynamics by co-transcriptional histone methylations
}

\author{
Hyeonju Woo ${ }^{1,2,5}$, So Dam Ha ${ }^{1,2,5}$, Sung Bae Lee ${ }^{3}$, Stephen Buratowski ${ }^{4}$ and TaeSoo Kim ${ }^{1,2}$
}

Co-transcriptional methylations of histone $\mathrm{H} 3$ at lysines 4 and 36, highly conserved methyl marks from yeast to humans, have profound roles in regulation of histone acetylation. These modifications function to recruit and/or activate distinct histone acetyltransferases (HATs) or histone deacetylases (HDACs). Whereas H3K4me3 increases acetylation at promoters via multiple HATs, H3K4me2 targets Set3 HDAC to deacetylate histones in 5' transcribed regions. In 3' regions of genes, H3K36me2/3 facilitates deacetylation by Rpd3S HDAC and slows elongation. Despite their important functions in deacetylation, no strong effects on global gene expression have been seen under optimized or laboratory growth conditions. Instead, H3K4me2-Set3 HDAC and Set2-Rpd3S pathways primarily delay the kinetics of messenger RNA (mRNA) and long noncoding RNA (IncRNA) induction upon environmental changes. A majority of mRNA genes regulated by these pathways have an overlapping IncRNA transcription either from an upstream or an antisense promoter. Surprisingly, the distance between mRNA and IncRNA promoters seems to specify the repressive effects of the two pathways. Given that co-transcriptional methylations and acetylation have been linked to many cancers, studying their functions in a dynamic condition or during cancer progression will be much more important and help identify novel genes associated with cancers.

Experimental \& Molecular Medicine (2017) 49, e326; doi:10.1038/emm.2017.19; published online 28 April 2017

\section{INTRODUCTION}

Chromatin is a complex of eukaryotic DNA and histone proteins packaged within the cell and plays essential roles in many cellular processes including RNA polymerase II (RNA polII) transcription, DNA replication, and DNA damage response. ${ }^{1}$ The basic repeating unit of chromatin is the nucleosome consisting of a histone octamer containing four core histones, $\mathrm{H} 3, \mathrm{H} 4, \mathrm{H} 2 \mathrm{~A}$ and $\mathrm{H} 2 \mathrm{~B}$ and 147 base pairs of DNA. ${ }^{2}$ Nucleosomes directly block transcription by RNA polII and should be evicted or reorganized during RNA polII initiation and elongation. ${ }^{3}$

Post-translational modifications on histone tails including acetylation, methylation, phosphorylation and ubiquitination play important roles in eukaryotic RNA polII transcription. ${ }^{1,4}$ Histone acetylation can directly promote transcription by disrupting the interaction between DNA and histone proteins. This mark also acts as a binding site for bromodomain proteins associated with factors that regulate chromatin structure and transcription. ${ }^{1}$ Histone acetylation is dynamically regulated by the antagonistic functions of histone acetyltransferases (HATs) and histone deacetylases (HDACs). How HATs and HDACs control histone acetylation at specific regions of a genome remains to be completely understood, but recent studies propose that site-specific histone methylations may affect local histone acetylation by targeting HATs or HDACs or stimulating their activities. ${ }^{4,5}$ Histone ubiquitination also regulates histone acetylation at a specific genomic position via H3K4 and H3K79 methylation. ${ }^{6,7}$

Although chromatin factors have been thought to be essential for global gene regulation, a recent study revealed a highly specific effect. Many chromatin regulators had little or no detectable effect on global gene expression in a steady state growth condition. ${ }^{8}$ Instead, single gene studies revealed that many chromatin regulators primarily modulated the kinetics of gene induction without changes in basal or final levels of expression. $^{9-11}$ Furthermore, loss of chromatin regulators resulted in greater effects on global gene expression during activation or repression upon environmental changes. ${ }^{12}$ Therefore, unlike gene-specific transcriptional activators or repressors turning on or off their target genes, chromatin regulators

\footnotetext{
${ }^{1}$ Department of Life Science, Ewha Womans University, Seoul, Korea; ${ }^{2}$ The Research Center for Cellular Homeostasis, Ewha Womans University, Seoul, Korea; ${ }^{3}$ Department of Brain and Cognitive Sciences, DGIST, Daegu, Republic of Korea and ${ }^{4}$ Department of Biological Chemistry and Molecular Pharmacology, Harvard Medical School, Boston, MA, USA

${ }^{5}$ These authors contributed equally to this work.

Correspondence: Professor T Kim, Department of Life Science, Ewha Womans University, Seoul 03760, Korea.
}

E-mail: tskim@ewha.ac.kr

Received 6 December 2016; accepted 20 December 2016 
can function as a transcriptional modulator that controls the kinetics of gene induction or repression. In addition, long noncoding RNA (lncRNA) transcription and lncRNA itself also play critical roles in gene regulation. ${ }^{13}$ Interestingly, $\sim 12 \%$ of yeast genes are overlapped with lncRNA transcription. ${ }^{14}$ A majority of these genes tend to be inducible suggesting the importance of overlapping lncRNA transcription in regulation of gene expression dynamics.

In natural growth conditions, cells must reprogram their gene expression patterns to adapt to rapidly changing environmental conditions. Fine tuning of gene expression dynamics by chromatin regulators can coordinate cellular processes to cell adaptation and fitness upon environmental changes. Here we review recent findings on modulation of gene expression dynamics by co-transcriptional methylations, their downstream effectors and overlapping lncRNA transcription.

\section{REGULATION OF CO-TRANSCRIPTIONAL METHYLATIONS OF H3K4 AND H3K36}

Histone methylations can be targeted by multiple distinct mechanisms. ${ }^{1}$ Factors involved in transcription repression contribute to recruitment of repressive histone methyltransferases (HMTs) for $\mathrm{H} 3 \mathrm{~K} 9$ or $\mathrm{H} 3 \mathrm{~K} 27$ methylation. In contrast, methylation of histone $\mathrm{H} 3$ at lysines 4 or 36 correlates positively with RNA polII transcription rate, and HMTs for these methyl marks interact with elongating RNA polII to localize histone methylations in transcribed regions. ${ }^{4,5,15-17}$

During transcription, the C-terminal domain (CTD) of Rpb1, the RNA polII largest subunit, is differentially phosphorylated by at least two kinases (Figure 1a). ${ }^{18}$ Whereas phosphorylation of the CTD repeat heptad sequence on serine 5 by Kin28, a subunit of TFIIH, peaks in transcription start site and $5^{\prime}$ transcribed regions, Ctk1 phosphorylates serine 2 of CTD in $3^{\prime}$ regions. ${ }^{18}$ CTD phosphorylated at serine 5 plays important roles in initiation and early elongation of transcription. This modification mediates a direct interaction between messenger RNA (mRNA) capping enzyme and RNA polII. ${ }^{18}$ Furthermore, transcription termination of snRNAs and short mRNA genes by Nrd1-exosome is stimulated via the interaction between $\mathrm{Nrd} 1$ and CTD phosphorylated at serine 5 (Figure 1a). Phosphorylation on serine 2 by Ctk1 also plays important roles during transcription elongation and termination. ${ }^{18}$ Many transcription elongation factors including Spt6 interact with CTD phosphorylated at serine 2 to stimulate elongation. This CTD phosphorylation is also important for co-transcriptional RNA splicing and transcription termination (Figure 1a). ${ }^{18}$

In addition, CTD phosphorylation also generates distinct patterns of histone $\mathrm{H} 3$ methylation at lysines 4 and 36 (Figure 1a). ${ }^{5}$ Serine 5 phosphorylation is known to stimulate H3K4 methylation by Set1-COMPASS in $5^{\prime}$ transcribed regions. ${ }^{19}$ Although a direct interaction between phosphorylated CTD at serine 5 and Set1-COMPASS has not been reported yet, inactivation of Kin28 reduced Set1 crosslinking. ${ }^{19}$ In budding yeast, Set1 is the sole methyltransferase for all states of $\mathrm{H} 3 \mathrm{~K} 4$ methylation. Whereas $\mathrm{H} 3 \mathrm{~K} 4 \mathrm{me} 3$ and Set1 crosslinking are observed at transcription initiation sites, $\mathrm{H} 3 \mathrm{~K} 4 \mathrm{me} 2$ and $\mathrm{H} 3 \mathrm{~K} 4 \mathrm{me} 1$ peak in $5^{\prime}$ and $3^{\prime}$ transcribed regions, respectively. ${ }^{15,16,19,20}$ The factors that affect the gradients of $\mathrm{H} 3 \mathrm{~K} 4$ methylation need to be determined. It is possible that Set1 activity/stability or composition of COMPASS may be altered during transcription elongation. ${ }^{21}$ Alternatively, Rad6-Bre1 may affect the distribution of $\mathrm{H} 3 \mathrm{~K} 4$ methylation. $\mathrm{H} 2 \mathrm{~B}$ ubiquitination on K123 by Rad6-Bre1 is required for H3K4me3 and H3K4me2 (Figure 1b). ${ }^{7,22-24}$ Enrichment of $\mathrm{H} 2 \mathrm{~B}$ ubiquitination in $5^{\prime}$ transcribed regions where $\mathrm{H} 3 \mathrm{~K} 4 \mathrm{me} 3$ and $\mathrm{H} 3 \mathrm{~K} 4 \mathrm{me} 2$ peak suggests that dissociation of this complex during elongation may result in peaks of $\mathrm{H} 3 \mathrm{~K} 4 \mathrm{mel}$ in $3^{\prime}$ ends of genes. ${ }^{25}$ In higher eukaryotes, multiple H3K4 HMTs including Set1A, Set1B and MLL1-4 proteins have been identified. ${ }^{26,27}$ Whereas Set1A and Set1B seem to be major HMTs for global H3K4 methylation, MLL3/4 targets H3K4me1 to enhancer regions suggesting the distinct functions of these H3K4 HMTs. ${ }^{26}$

The H3K36 HMT, Set2 preferentially binds to phosphorylated CTD at both serine 5 and serine 2 and targets $\mathrm{H} 3 \mathrm{~K} 36 \mathrm{me} 2 / 3$ within the body of genes (Figure 1a). ${ }^{28-31}$ Mutation in the CTD interacting domain of Set 2 or deletion of CTK1 results in loss of Set 2 crosslinking and defects of H3K36me2/3. ${ }^{29}$ Factors involved in transcription elongation also affect H3K36 methylation (Figure 1c). Deletion of CHD1 chromatin remodeler causes a shift of $\mathrm{H} 3 \mathrm{~K} 36 \mathrm{me} 3$ to $5^{\prime}$ transcribed regions and Asf1 histone chaperone stimulates H3K36me3 and Set2 crosslinking. ${ }^{32,33}$ In addition to CTD binding, the $\mathrm{N}$-terminal region of Set 2 interacts with histone $\mathrm{H} 4$ to stimulate $\mathrm{H} 3 \mathrm{~K} 36 \mathrm{me} 2 / \mathrm{me} 3 .^{34}$

Although histone methylation was originally thought to be a permanent mark, enzymes that can reverse histone methylation have been identified. Mammalian lysine-specific demethylase 1 (LSD1) is the first enzyme discovered that demethylates $\mathrm{H} 3 \mathrm{~K} 4 \mathrm{me} 2$ and $\mathrm{H} 3 \mathrm{~K} 4 \mathrm{me} 1$, but not $\mathrm{H} 3 \mathrm{~K} 4 \mathrm{me} 3 .{ }^{35}$ A second class of demethylases found from bacteria to humans, the Jmjc domain proteins can demethylate all three lysine methylation states and broad ranges of substrates. ${ }^{36}$ Antagonistic functions of HMTs and histone demethylases (HDMs) may maintain optimal levels of histone $\mathrm{H} 3$ methylation at lysines 4 or 36 (Figures $1 \mathrm{~b}$ and $\mathrm{c}$ ). ${ }^{37,38}$

\section{THE DOWNSTREAM EFFECTORS OF CO-TRANSCRIP- TIONAL H3 METHYLATIONS}

Unlike acetylation, histone methylation is not known to directly affect chromatin structure. Instead, site-specific methylations act as binding sites for various downstream effector proteins containing chromodomains, tudor domains, PHD fingers and PWWP domains. ${ }^{1,4}$ These effector proteins are often found in large protein complexes including HATs, HDACs or chromatin remodeling complexes. ${ }^{6}$ The interaction between a site-specific methylation and an effector protein can regulate local chromatin structure and RNA polII transcription.

The interaction between Set1-COMPASS and RNA polII and the correlation between $\mathrm{H} 3 \mathrm{~K} 4 \mathrm{me} 3$ and transcription rate suggest a positive role of this methyl mark in 
a

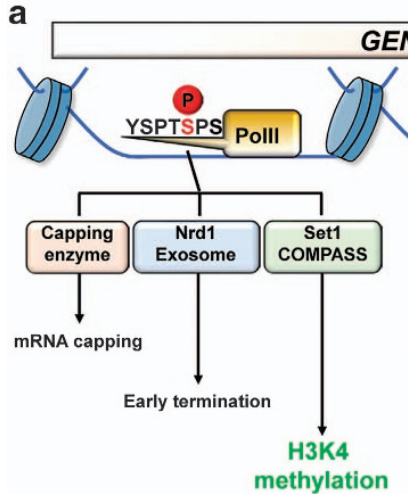

b

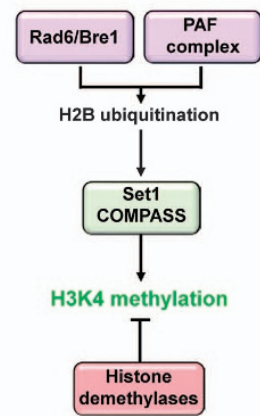

Figure 1 Model for regulation of co-transcriptional methylations. (a) At an early stage of transcription, C-terminal domain (CTD) phosphorylation at serine 5 recruits messenger RNA (mRNA) capping machinery and early termination factors. This modification also contributes to recruitment of Set1-COMPASS that methylates histone $\mathrm{H} 3$ at lysine 4 in $5^{\prime}$ transcribed regions. During transcription elongation, CTD phosphorylation on serine 2 functions to recruit mRNA splicing machinery and termination factors. In addition, serine 2 phosphorylation of CTD and low levels of serine 5 phosphorylation also create a binding site for Set2 histone methyltransferase (HMT) that methylates H3K36 in $3^{\prime}$ transcribed regions. (b, c) Factors that influence $\mathrm{H} 3 \mathrm{~K} 4$ and $\mathrm{H} 3 \mathrm{~K} 36$ methylation patterns.

transcription. ${ }^{15-17,19,20}$ Many proteins that stimulate RNA polII transcription can bind $\mathrm{H} 3 \mathrm{~K} 4 \mathrm{me} 3$. In budding yeast, Yng1, Yng2 and Sgf29 are associated with distinct HATs, and these were shown to strongly bind H3K4me3. ${ }^{39,40}$ Yng1 and Yng2 are PHD proteins and the yeast homologs of the ING (INhibitor of Growth) gene family that may function as tumor suppressors. ${ }^{41}$ Yng1 is a component of NuA3 HAT, which preferentially acetylates histone $\mathrm{H} 3$ at $\mathrm{K} 14{ }^{42-44} \mathrm{Yng} 2$ is a subunit of NuA4, a major HAT for histone H4. ${ }^{45}$ The Sgf29 tudor domain protein promotes chromatin binding of SAGA HAT and histone $\mathrm{H} 3$ acetylation (Figure 2a). ${ }^{40}$ In higher eukaryotes, the TAF3 subunit of TFIID has a PHD finger that directly binds $\mathrm{H} 3 \mathrm{~K} 4 \mathrm{me} 3$ to promote RNA polII initiation. ${ }^{46}$ Furthermore, the interaction between $\mathrm{H} 3 \mathrm{~K} 4 \mathrm{me} 3$ and ING4/ ING5 PHD fingers promotes histone $\mathrm{H} 3$ acetylation by HBO1 HAT (Figure 2a). ${ }^{47,48}$ Interestingly, some PHD finger proteins associated with transcriptional corepressors also bind H3K4me3. In yeast, Pho23 and Rxt1, both components of Rpd3 large(Rpd3L) HDAC, directly bind to H3K4me3 via their
PHD fingers. ${ }^{39,49-51}$ Mammalian ING2, a mSin3a-HDAC1 complex subunit also preferentially binds to $\mathrm{H} 3 \mathrm{~K} 4 \mathrm{me} 3$ to promote gene repression (Figure $2 \mathrm{a}$ ). ${ }^{52}$

The H3K4me2 peak in $5^{\prime}$ transcribed regions can be recognized by several factors, including Set3, Phf7 and nardilysin, that regulate transcription and histone acetylation (Figure 2b). ${ }^{6,53,54}$ Set3 is a component of Set3 HDAC, which also contains two deactylase subunits, Hos2 and Hst1. ${ }^{55}$ The Set3 PHD finger preferentially binds H3K4me2 and targets Set3 HDAC to $5^{\prime}$ transcribed regions. ${ }^{6}$

H3K36 methylation by Set2 also creates a binding site for various factors involved in histone acetylation/deacetylation and transcription elongation (Figure 2c). In yeast, the Eaf3 chromodomain, Nto1 PHD finger, Pdp3 PWWP domain and Ioc4 PWWP domain are known to bind H3K36me3. 39,50,51,56,57 Eaf3 is found in both Rpd3 small(Rpd3S) HDAC and NuA4 HAT. ${ }^{50,51,58}$ Both Not1 and Pdp3 are components of NuA3 HAT. ${ }^{39,56}$ These findings suggest both a positive and negative role of $\mathrm{H} 3 \mathrm{~K} 36 \mathrm{me} 3$ in histone acetylation. The interaction between Ioc4 PWWP domain and H3K36me3 helps maintain chromatin integrity during RNA polII elongation (Figure 2c). ${ }^{57}$

Both $\mathrm{H} 3 \mathrm{~K} 4$ and $\mathrm{H} 3 \mathrm{~K} 36$ co-transcriptional methylations may also inhibit binding of some factors to histones or transcribed regions. BHC80 PHD finger binds unmethylated H3K4 and this binding is dramatically inhibited by $\mathrm{H} 3 \mathrm{~K} 4$ methylation. ${ }^{59}$ Crosslinking of histone chaperones Spt6 and Asf1 to coding regions is inhibited by $\mathrm{H} 3 \mathrm{~K} 36$ methylation. ${ }^{60}$ The functions of each individual downstream effector for $\mathrm{H} 3 \mathrm{~K} 4$ or $\mathrm{H} 3 \mathrm{~K} 36$ methylation remain to be completely understood.

\section{DEACETYLATION BY H3K4ME2-SET3 HDAC PATHWAY DELAYS GENE INDUCTION}

The Set3 HDAC consists of seven polypeptides including two histone deacetylases, Hos 2 and Hst $1 .{ }^{55}$ The Set3 protein has both a SET domain and a PHD finger. ${ }^{55}$ Whereas the function of the Set3 SET domain needs to be determined, the Set3 PHD finger has been shown to preferentially bind H3K4me2 peaking from $5^{\prime}$ to the middle parts of genes. ${ }^{6,39}$ Loss of Set3 HDAC increased acetylation in $5^{\prime}$ transcribed regions (Figure 3a). ${ }^{6}$ Elimination of $\mathrm{H} 3 \mathrm{~K} 4 \mathrm{me} 2$ and $\mathrm{H} 3 \mathrm{~K} 4 \mathrm{me} 3$ by deleting SET1 or Rad6-Bre1 H2B ubiquitination pathway also resulted in hyperacetylation in $5^{\prime}$ regions. ${ }^{6}$ Furthermore, mutation in the Set3 PHD finger that abrogates histone binding also increased acetylation, indicating that the interaction between $\mathrm{H} 3 \mathrm{~K} 4 \mathrm{me} 2$ and the Set3 PHD finger maintains hypoacetylation in $5^{\prime}$ transcribed regions. ${ }^{6}$ Deacetylation by Set3 HDAC may suppress spreading of acetylation into gene bodies.

Interestingly, although loss of Set3 HDAC significantly changed histone acetylation, no difference in transcript levels was observed between cells containing or lacking Set3 under steady-state growth conditions. ${ }^{6}$ A genome-wide transcript profiling of cells grown in synthetic complete media containing glucose also revealed no detectable changes in global transcript levels upon deletion of Set3 HDAC. ${ }^{8}$ Interestingly, the effects of Set3 HDAC only became apparent when gene expression is 


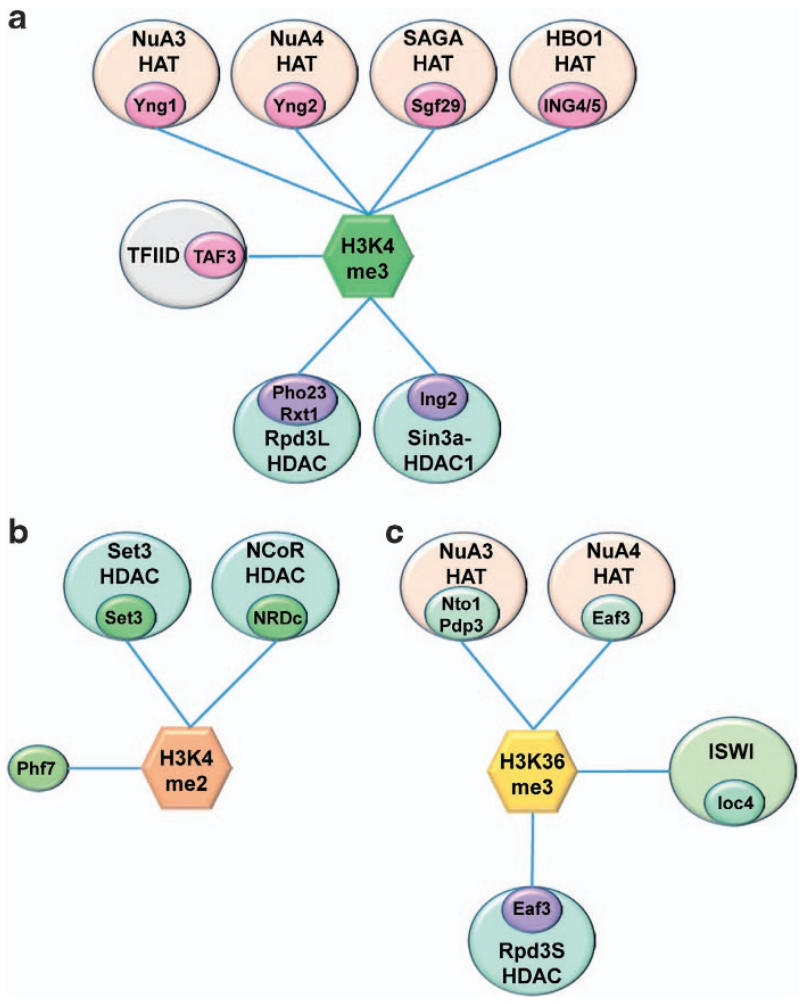

Figure 2 The downstream readers of co-transcriptional methylations. Multiple factors that positively and negatively affect gene expression bind co-transcriptional histone methylation of H3K4 and H3K36. (a) A distinct subunit (indicated by smaller circles) from multiple histone acetyltransferases (HATs) including NuA3, NuA4, SAGA and HBO1 HATs and TFIID complex binds H3K4me3. In addition, histone deacetylases (HDACs), Rpd3L in yeast and Sin3a-HDAC1 in mammals also interact with H3K4me3 via their specific subunits. (b) Factors that influence transcription bind to H3K4me2. The Set3 PHD finger and NRDc associated with HDACs preferentially bind $\mathrm{H} 3 \mathrm{~K} 4 \mathrm{me} 2$. (c) Chromatin regulators that interact with H3K36me3. Two PHD finger subunits, Nto1 and Pdp3 of NuA3 HAT and Eaf3 chromodomain of both NuA4 HAT and Rpd3S HDAC interact with H3K36me3. The PWWP domain of loc4, a subunit of chromatin remodeler, ISW1 also binds H3K36me3.

dynamically changed by altering growth conditions. Histone deacetylation is generally associated with gene repression, but Set3 HDAC seems to be both repressive and activating. Set3 HDAC is required for proper induction kinetics of GAL1 and INO1 genes. ${ }^{6,61}$ Also, this complex negatively regulates the induction of meiotic genes upon nitrogen starvation. ${ }^{55}$ Molecular mechanisms for how Set3 HDAC differentially controls these genes have been partially understood.

We recently showed that Set3 HDAC delays the induction of $\sim 120$ genes during carbon source shifts. ${ }^{62}$ Steady-state levels of transcripts were almost the same in wild type and a mutant for Set3. However, a stronger or more rapid induction of these target genes was seen in SET3 deletion cells during carbon source shifts (Figure 3b). 12,62 Two histone deacetylases, Hos2 and Hst1 were also required for Set3-mediated delay of gene induction. ${ }^{62}$ Surprisingly, although Set3 HDAC preferentially affects histone acetylation in $5^{\prime}$ regions of mRNA genes where H3K4me2 peaks, Set3-repressed genes showed increased acetylation at promoter regions in SET3 deletion cells. ${ }^{6,62}$ These results suggest that the Set3-affected genes may have non-canonical patterns of histone methylation. Analysis of histone methylation pattern revealed that $~ 53 \%$ of Set 3 target genes had high levels of H3K4me2 at transcription start sites (Figure 3b). ${ }^{62}$ Apparently, this pattern of $\mathrm{H} 3 \mathrm{~K} 4 \mathrm{me} 2$ was established by overlapping lncRNA transcription. A majority of Set3-regulated genes were overlapped by a lncRNA transcription either from a distal or an antisense promoter. ${ }^{62}$ These lncRNA promoters were enriched with $\mathrm{H} 3 \mathrm{~K} 4 \mathrm{me} 3$ but the associated Set3-regulated mRNA promoters had high levels of H3K4me2 (Figure 3b). At these genes, Set3 HDAC affected histone acetylation only at mRNA promoters but not at lncRNA promoters. These findings suggest that targeting of H3K4me2 and Set3 HDAC to mRNA promoters via lncRNA transcription delays or fine tunes gene induction upon environmental changes.

An additional function of $\mathrm{H} 3 \mathrm{~K} 4 \mathrm{me} 2-\mathrm{Set} 3 \mathrm{HDAC}$ is to repress lncRNA transcription from cryptic promoters within $5^{\prime}$ transcribed regions. Although deletion of SET3 had no effect on mRNA transcription at these genes, the overlapped cryptic promoters were much more active in this mutant during carbon source shifts (Figure 3b). Therefore, Set3 HDAC maintains optimal expression dynamics of both mRNA and lncRNA.

\section{THE SET2-RPD3S HDAC PATHWAY SLOWS mRNA AND IncRNA INDUCTION}

H3K36 methylation by Set2 plays important functions during transcription elongation. Although a direct binding of Set2 to elongating RNA polII and the correlation of $\mathrm{H} 3 \mathrm{~K} 36 \mathrm{me} 3$ with transcription rate suggested a positive function in RNA polII transcription, loss of Set2 showed phenotypes associated with transcription inhibition. Deletion of SET2 or mutation of H3K36 to A or R bypassed loss of Bur1, a positive elongation factor. ${ }^{50,63}$ Mutations of other elongation factors such as Spt16 and Spt5 were also suppressed by loss of Set2.64,65 Furthermore, although many elongation mutants are sensitive to 6-AU or MPA that inhibits elongation, SET2 deleting cells are resistant to these chemicals. ${ }^{30,50}$

During transcription elongation, histones are thought to be acetylated by one or more HATs to promote elongation on the chromatin template. Once RNA polII passes through the gene, deacetylation is important to re-establish a repressive chromatin configuration that blocks aberrant transcription. Set2-mediated H3K36 methylation facilitates histone deacetylation by Rpd3S within coding regions (Figure 4a). ${ }^{50,51,58,66,67}$ Two subunits, Eaf3 and Rco1, appear to be important for chromatin binding of this complex. Eaf3 chromodomain directly binds to methylated histones on $\mathrm{H} 3 \mathrm{~K} 36$ and Rcol PHD finger interacts with unmodified histone tails. ${ }^{39,68-70}$ Rpd3S seems to be also recruited to transcribed regions by elongating RNA polII via interaction with the phosphorylated 

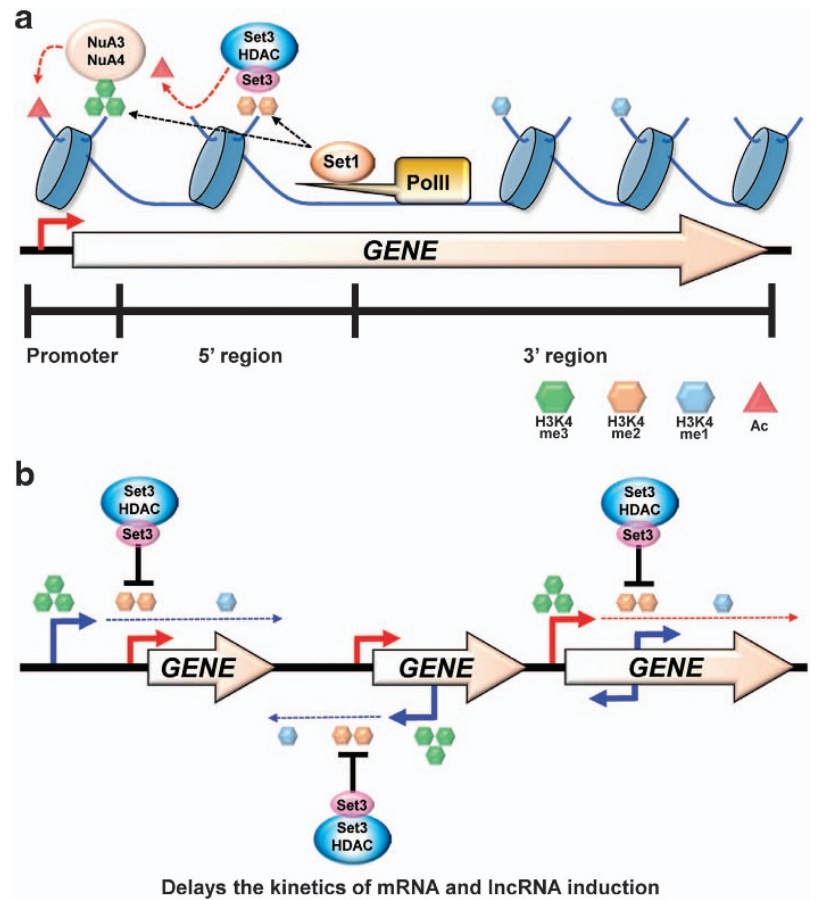

Figure 3 Model for regulation of histone acetylation and gene induction by Set3 HDAC. (a) The C-terminal domain (CTD)-interacting Set1 HMT deposits H3K4me3 and H3K4me2 to promoters and $5^{\prime}$ transcribed regions, respectively. NuA3 and NuA4 HATs may acetylate histones at promoters via the interaction between PHD finger proteins, Yng1 and Yng2 and H3K4me3. In $5^{\prime}$ transcribed regions, the Set3 PHD finger binds H3K4me2 and two histone deacetylases (HDACs), Hos2 and Hst1 deacetylate histones. (b) Long noncoding RNA (IncRNA) transcription from an upstream or an antisense promoter targets $\mathrm{H} 3 \mathrm{~K} 4 \mathrm{me} 2$ and Set3 HDAC to messenger RNA (mRNA) promoters. Deacetylation by Set3 HDAC delays gene induction upon environmental changes. Transcription from the mRNA promoter of a gene places $\mathrm{H} 3 \mathrm{~K} 4 \mathrm{me} 2$ to $5^{\prime}$ transcribed regions and Set3 HDAC slows the kinetics of IncRNA induction.

CTD, but histone deacetylation requires a direct interaction of this complex with methylated histones on H3K36. ${ }^{66,67}$ Deacetylation by Rpd3S within coding regions slows RNA polII elongation (Figure 4a). ${ }^{50}$ The Set2-Rpd3S pathway also contributes to repress cryptic promoters that produce sense or antisense lncRNAs. Many transcription elongation factors and chromatin regulators including Spt6, Spt16 and Ctk1 suppress internal initiation from cryptic promoters within open reading frames. ${ }^{71}$ Loss of the Set2-Rpd3S pathway resulted in hyperacetylation within coding regions and initiation of RNA polII transcription from cryptic promoters. ${ }^{51,72}$ A recent study also identified a novel class of antisense transcripts repressed by Set $2 .{ }^{73}$

Although previous studies on the Set2-Rpd3S pathway elucidated molecular mechanisms for how the pathway is targeted and inhibits lncRNA transcription from cryptic promoters, its function in mRNA gene regulation remains unclear. Surprisingly, despite its importance in suppression of cryptic promoters via histone deacetylation, global mRNA transcript levels were not affected by loss of this pathway under a steady-state growth condition. ${ }^{8}$ To further explore the exact function of the Set2-Rpd3S pathway, we recently monitored genome-wide transcript levels in wild type and SET2 deletion cells by high-resolution, strand-specific tiling arrays during carbon source shifts. ${ }^{74}$ Although steady-state levels of transcripts were not affected, $\sim 80$ genes showed a strong and more rapid induction upon SET2 deletion. ${ }^{74}$ These findings suggest that Set2-Rpd3S pathway is important for modulating mRNA expression dynamics during environmental changes (Figure 4b).

Analysis of Set2-affected genes revealed that $69 \%$ of these genes had an overlapping lncRNA transcription from either an upstream or an antisense promoter. ${ }^{74}$ One class of Set2-repressed genes was overlapped with lncRNA transcription from a distal promoter (Figure $4 \mathrm{~b}$ ). AAD10 is one of these Set2-repressed genes, and is overlapped by sense IncRNA transcription from the promoter of upstream gene YJR154W. Although $A A D 10$ lncRNA was detected in rich media, neither Aad10 nor Yjr154w protein was observed, suggesting that this lncRNA may not be translated into a protein. This locus showed an interesting pattern of co-transcriptional histone methylation. Although $\mathrm{H} 3 \mathrm{~K} 4 \mathrm{me} 3$ and $\mathrm{H} 3 \mathrm{~K} 36 \mathrm{me} 3$ are in general enriched at promoters and $3^{\prime}$ part of genes, respectively, the $A A D 10$ promoter showed high levels of H3K36me3 but not H3K4me3 (Figure 4b). ${ }^{74}$

The overlapping transcription at the majority of Set2regulated genes came from downstream antisense promoters (Figure 4b). For example, the SUL1 gene was overlapped by the antisense lncRNA SUT452, which is constitutively transcribed. H3K4me3 was highest at SUT452 promoter near the $3^{\prime}$ end of SUL1, while the SUL1 mRNA promoter had high levels of H3K36me3. ${ }^{74}$ We revealed that H3K36me3 deposited during IncRNA transcription facilitated histone deacetylation of the overlapped mRNA promoter by Rpd3S. ${ }^{74}$ Loss of Set2 or subunits of Rpd3S showed increased acetylation at $A A D 10$ and SUL1 promoters but not at YJR154W or SUT452 promoters. Accordingly, AAD10 and SUL1 transcripts but not the associated lncRNAs were strongly induced in mutants for the Set2-Rpd3S pathway during carbon source shifts. ${ }^{74}$

A previous study reported that although 1685 genes showed increased acetylation within open reading frames in SET2 deleting cells, only half of them produced internal lncRNA transcripts. $^{72}$ It is possible that any lncRNA transcripts produced from these genes are not observed because they can be rapidly degraded by nucleases such as the nuclear exosome. For example, both antisense and short sense transcripts were seen at STE11 and CTT1 in double mutants lacking both Rpd3S ( $\operatorname{rcol} \Delta)$ and nuclear exosome $(\operatorname{rrp} 6 \Delta)$, but only the short sense transcripts were seen upon deletion of RCO1 alone. $^{75}$ These suggest that antisense transcripts from STE11 and CTT1 are rapidly degraded by nuclear exosome.

Another interesting possibility is that cryptic promoters within genes may be inducible and therefore active only under certain conditions. Previous studies revealed that some cryptic 

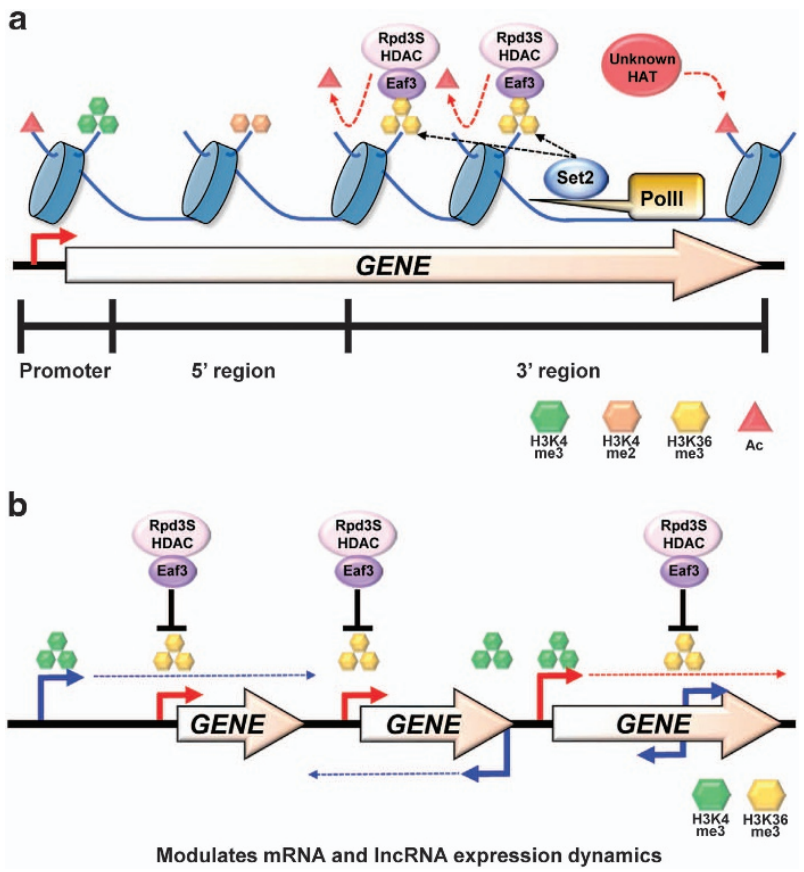

Figure 4 Model for modulation of acetylation and gene induction by the Set2-Rpd3S pathway. (a) Set2 HMT interacts with elongating RNA pollI and methylates on H3K36 in $3^{\prime}$ transcribed regions. Eaf3 chromodomain binds H3K36me2/3 and Rco1 PHD finger interacts with histone tails to stabilize chromatin binding of Rpd3S. Rpd3 deacetylates histones to slow elongation and suppress cryptic promoters. (b) IncRNA transcription from an upstream or an antisense promoter targets $\mathrm{H} 3 \mathrm{~K} 36 \mathrm{me} 3$ and Rpd3S HDAC to mRNA promoters. Deacetylation at mRNA promoters by Rpd3S slows gene induction. Transcription from the mRNA promoter of a gene localizes H3K36me3 to inducible cryptic promoters. Deacetylation by Rpd3S histone deacetylase (HDAC) delays the rate of IncRNA induction. HMT, histone methyltransferase; IncRNA, long noncoding RNA; mRNA, messenger RNA; RNA polII, RNA polymerase II.

transcripts were induced by nutritional shifts. ${ }^{62,71}$ We identified 118 internal cryptic promoters generating a short sense transcript and 639 producing an antisense transcript in SET2 deletion cells during carbon source shifts. ${ }^{74}$ Surprisingly, 416 of them were induced by specific carbon sources (Figure $4 \mathrm{~b}$ ). Furthermore, three cryptic promoters in the PCA1 gene that are repressed by Set2 differently responded to nutritional shifts. ${ }^{74}$ One was constitutively active but the other two were induced or repressed during carbon source shifts. These results suggest that in addition to mRNA genes, the Set2-Rpd3S pathway can delay the induction of lncRNAs upon environmental changes.

\section{GENE REGULATION BY OVERLAPPING IncRNA TRANSCRIPTION}

Two recent studies from our group have revealed that H3K4me2-Set3 HDAC and the Set2-Rpd3S pathway modulate the induction of mostly distinct target genes. ${ }^{62,74}$ However, the genes regulated by both pathways have a common feature. A majority of them are overlapped by lncRNA transcription either from a distal or an antisense promoter. The overlapping lncRNA transcription, rather than the lncRNA itself, may target H3K4me2 or H3K36me3 to promoter regions of distinct genes and delay the kinetics of gene induction.

DCI1 is one of the Set3-repressed genes overlapped by lncRNA transcription from a distal promoter (Figure 5). Upon SET3 deletion, lncRNA transcript levels were not changed, but DCI1 mRNA transcripts were elevated during galactose incubation. Before induction, the DCI1 mRNA promoter had a peak of H3K4me2 as a result of the overlapping lncRNA transcription. The DCI1 promoter showed increased acetylation upon loss of Set3, explaining its elevated transcription upon induction..$^{62}$ No derepression of this gene was observed in mutants for the Set2-Rpd3S pathway. In contrast, $A A D 10$ is also overlapped with lncRNA transcription from an upstream promoter, but is repressed by Set2 (Figure 5). Interestingly, the $A A D 10$ promoter was enriched with $\mathrm{H} 3 \mathrm{~K} 36 \mathrm{me} 3$ and derepressed in mutants for the Set2-Rpd3S pathway but not Set3C.

What determines the specificity for these two pathways? An interesting difference between Set3- and Set2-repressed genes is the distance between mRNA and IncRNA promoters (Figure 5). For example, the lncRNA and mRNA promoters of the DCI1 are $\sim 0.55 \mathrm{~kb}$ apart, but the distance between the two $A A D 10$ promoters is $\sim 1.5 \mathrm{~kb}$. Furthermore, the average distances between the lncRNA and mRNA promoters of Set3-repressed and Set2-regulated genes were $\sim 0.9 \mathrm{~kb}$ and $2.0 \mathrm{~kb}$, respectively. These findings support the idea that the distance between mRNA and lncRNA promoter may specify the Set2-Rpd3S or Set3 HDAC-mediated repression. ${ }^{74}$

Consistent with this, reducing the distance between the two promoters of $A A D 10$ caused expression to become insensitive to the Set2-Rpd3S pathway. H3K36me3 was significantly reduced at the AAD10 mRNA promoter and histone acetylation was not changed in mutants for the Set2-Rpd3S pathway. In addition, $A A D 10$ transcript levels were very similar in both wild type and SET2 deletion cells. ${ }^{74}$ These results indicate that shortening the distance between the two promoters of $A A D 10$ alleviates repression by the Set2-Rpd3S pathway. Furthermore, although $\mathrm{H} 3 \mathrm{~K} 4 \mathrm{me} 2$ was low at $A A D 10$ promoter in the normal context, an elevated level was seen when the distance was reduced. Correspondingly, histone acetylation and AAD10 transcript levels were now increased in mutants for Set3 HDAC. Thus, alteration of the distance between the two promoters switches the repression of $A A D 10$ by H3K36me3Rpd3S to the H3K4me2-Set3 HDAC repression pathway.

Transcription of lncRNA as well as lncRNA itself can play important roles in gene regulation. ${ }^{13}$ Our recent findings suggest that although lncRNA can be transcribed from any site of a genome, positions of overlapping lncRNA promoters may be important for targeting distinct histone methylations and chromatin regulators to promoter regions of an associated mRNA gene.

\section{DISCUSSION}

Co-transcriptional histone methylations and their downstream effectors have critical roles in almost all steps of gene regulation 


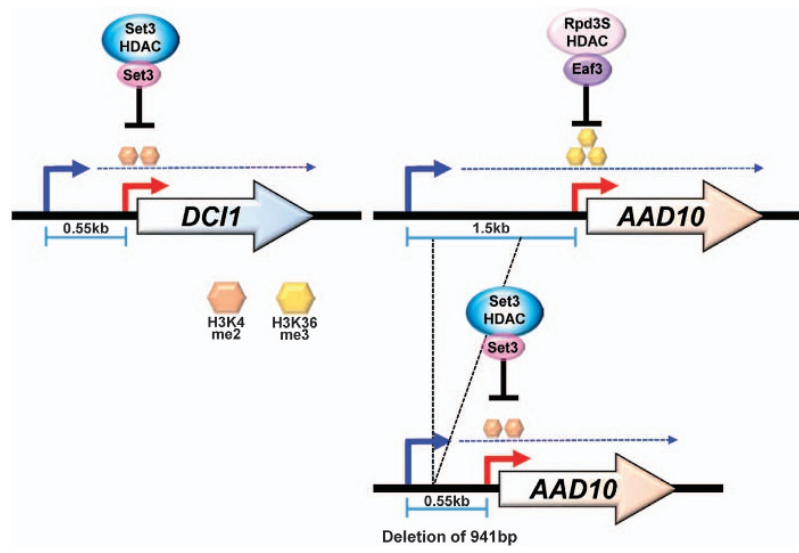

Figure 5 Model for regulation of gene induction by IncRNA transcription and two HDACs. Set3 HDAC and the Set2-Rpd3S pathway negatively regulate the kinetics of $A A D 10$ and $D C / 1$ induction, respectively. $\mathrm{H} 3 \mathrm{~K} 4 \mathrm{me} 2$ and Set3 HDAC are targeted to DCI1 mRNA promoter via an overlapping IncRNA transcription and slow $D C / 1$ induction. In contrast, IncRNA transcription from an upstream promoter of $A A D 10$ targets $\mathrm{H} 3 \mathrm{~K} 36 \mathrm{me} 3$ and Rpd3S to $A A D 10$ mRNA promoter. Surprisingly, shortening the distance between the two promoters of $A A D 10$ replaces $\mathrm{H} 3 \mathrm{~K} 36$ me3-Rpd3S with $\mathrm{H} 3 \mathrm{~K} 4 \mathrm{me} 2-\mathrm{Set} 3 \mathrm{HDAC}$ for $A A D 10$ repression. Thus, position of the IncRNA promoter may be important for specifying the repressive effects of the two HDACs. HDAC, histone deacetylase; mRNA, messenger RNA; IncRNA, long noncoding RNA.

including initiation, elongation, RNA processing and termination. Despite their importance, global gene expression is often not significantly affected by loss of these factors in optimized or laboratory growth conditions. Instead, stronger effects can be observed in more realistically dynamic conditions where gene expression is continuously changing. H3K4me2-Set3 HDAC and the Set2-Rpd3S pathways deacetylate histones at certain mRNA or lncRNA promoters where H3K4me2 and H3K36me3 are enriched, respectively, and negatively regulate the rate of mRNA or IncRNA induction. ${ }^{62,74}$ However, the HATs responsible for acetylation at these regions are not completely known. Some factors associated with distinct HATs have been shown to bind H3K36me3. For example, Nto1 and Pdp3 of NuA3 preferentially bind to H3K36me3. ${ }^{39,56}$ Furthermore, Eaf3 is a component of both NuA4 HAT and Rpd3S HDAC. Future works will determine if NuA3 or NuA4 HAT can acetylate histones at Set2-repressed promoters and increase the rate of mRNA and IncRNA induction upon environmental changes.

Regulation of gene expression dynamics is likely to be essential for normal development process, adaptation to new environmental conditions and proper cellular responses to extracellular stresses. A previous report revealed that knockdown of many chromatin regulators had no obvious effect on stem cell maintenance. ${ }^{76}$ Instead, greater effects are observed upon loss of chromatin regulators when cells are triggered with stresses or undergo cellular differentiation, suggesting that the maintenance of gene expression dynamics during this period is critical for fate decisions of stem cells. Future works need to identify the genes that are dynamically regulated during stem cell differentiation, and study the function of chromatin regulators in this process.

The factors involved in co-transcriptional histone methylations and their downstream effectors have been directly linked to many cancers. ${ }^{77-79}$ Altered expression, translocation, and amplification of genes for H3K4 HMTs and H3K36 HMTs have been reported in distinct types of cancers. ${ }^{78,79}$ Overexpression or mutations of genes encoding HDMs for both methyl marks are also observed in cancers. ${ }^{77,79}$ In addition, ING family proteins that directly bind methylated lysines function as tumor suppressors or oncogenes. ${ }^{48,80}$ Current studies tend to directly compare global gene expression in normal and cancer cells to identify cancer-associated genes. However, since mutation or overexpression of the factors related to co-transcriptional methylations will have greater effects on gene expression changes in a more dynamic condition, studying their functions during cancer development and progression will be much more important and allow us to identify novel genes that are differentially expressed in cancer cells.

\section{CONFLICT OF INTEREST}

The authors declare no conflict of interest.

\section{ACKNOWLEDGEMENTS}

This research was supported by grants to TK (the Basic Science Research Program of the National Research Foundation of Korea (NRF) funded by the Ministry of Education, Science and Technology (NRF-2013R1A1A1008634 and NRF-2012R1A5A1048236), the National Research Foundation of Korea funded by Korean Government (NRF-2013S1A2A2035342)).

1 Kouzarides T. Chromatin modifications and their function. Cell 2007; 128 : 693-705.

2 Luger K, Mader AW, Richmond RK, Sargent DF, Richmond TJ. Crystal structure of the nucleosome core particle at 2.8A resolution. Nature 1997; 389: 251-260.

3 Workman JL, Kingston RE. Alteration of nucleosome structure as a mechanism of transcriptional regulation. Annu Rev Biochem 1998; 67: 545-579.

4 Li B, Carey M, Workman JL. The role of chromatin during transcription. Cell 2007; 128: 707-719.

5 Buratowski S, Kim T. The role of cotranscriptional histone methylations. Cold Spring Harb Symp Quant Biol 2010; 75: 95-102.

$6 \mathrm{Kim} \mathrm{T}$, Buratowski S. Dimethylation of H3K4 by Set1 recruits the Set3 histone deacetylase complex to $5^{\prime}$ transcribed regions. Cell 2009; 137: 259-272.

7 Kim J, Guermah M, McGinty RK, Lee JS, Tang Z, Milne TA et al. RAD6Mediated transcription-coupled $\mathrm{H} 2 \mathrm{~B}$ ubiquitylation directly stimulates H3K4 methylation in human cells. Cell 2009; 137: 459-471.

8 Lenstra TL, Benschop JJ, Kim T, Schulze JM, Brabers NA, Margaritis T et al. The specificity and topology of chromatin interaction pathways in yeast. Mol Cell 2011; 42: 536-549.

9 Barbaric S, Walker J, Schmid A, Svejstrup JQ, Horz W. Increasing the rate of chromatin remodeling and gene activation-a novel role for the histone acetyltransferase Gcn5. EMBO J 2001; 20: 4944-4951.

10 Korber P, Barbaric S, Luckenbach T, Schmid A, Schermer UJ, Blaschke D et al. The histone chaperone Asf 1 increases the rate of histone eviction at the yeast $\mathrm{PHO} 5$ and $\mathrm{PHO} 8$ promoters. J Biol Chem 2006; 281: $5539-5545$ 
11 Williams SK, Truong D, Tyler JK. Acetylation in the globular core of histone H3 on lysine-56 promotes chromatin disassembly during transcriptional activation. Proc Natl Acad Sci USA 2008; 105: 9000-9005.

12 Weiner A, Chen HV, Liu CL, Rahat A, Klien A, Soares L et al. Systematic dissection of roles for chromatin regulators in a yeast stress response. PLOS Biol 2012; 10: e1001369.

13 Pelechano V, Steinmetz LM. Gene regulation by antisense transcription. Nat Rev Genet 2013; 14: 880-893.

14 Xu Z, Wei W, Gagneur J, Clauder-Munster S, Smolik M, Huber W et al. Antisense expression increases gene expression variability and locus interdependency. Mol Syst Biol 2011; 7: 468

15 Pokholok DK, Harbison CT, Levine S, Cole M, Hannett NM, Lee TI et al. Genome-wide map of nucleosome acetylation and methylation in yeast. Cell 2005; 122: 517-527.

16 Liu CL, Kaplan T, Kim M, Buratowski S, Schreiber SL, Friedman N et al. Single-nucleosome mapping of histone modifications in S. cerevisiae. PLOS Biol 2005; 3: e328.

17 Barski A, Cuddapah S, Cui K, Roh TY, Schones DE, Wang Z et al. Highresolution profiling of histone methylations in the human genome. Cell 2007; 129: 823-837.

18 Buratowski S. Progression through the RNA polymerase II CTD cycle. Mol Cell 2009; 36: 541-546.

$19 \mathrm{Ng} \mathrm{HH}$, Robert F, Young RA, Struhl K. Targeted recruitment of Set1 histone methylase by elongating Pol II provides a localized mark and memory of recent transcriptional activity. Mol Cell 2003; 11: 709-719.

20 Kirmizis A, Santos-Rosa H, Penkett CJ, Singer MA, Vermeulen M, Mann M et al. Arginine methylation at histone H3R2 controls deposition of H3K4 trimethylation. Nature 2007: 449: 928-932.

21 Soares LM, Radman-Livaja M, Lin SG, Rando OJ, Buratowski S. Feedback control of Set1 protein levels is important for proper H3K4 methylation patterns. Cell Rep 2014; 6: 961-972.

22 Dehe PM, Pamblanco M, Luciano P, Lebrun R, Moinier D, Sendra R et al. Histone $\mathrm{H} 3$ lysine 4 mono-methylation does not require ubiquitination of histone H2B. J Mol Biol 2005; 353: 477-484.

23 Lee JS, Shukla A, Schneider J, Swanson SK, Washburn MP, Florens L et al. Histone crosstalk between $\mathrm{H} 2 \mathrm{~B}$ monoubiquitination and $\mathrm{H} 3$ methylation mediated by COMPASS. Cell 2007; 131: 1084-1096.

24 Vitaliano-Prunier A, Menant A, Hobeika M, Geli V, Gwizdek C, Dargemont C. Ubiquitylation of the COMPASS component Swd2 links H2B ubiquitylation to H3K4 trimethylation. Nat Cell Biol 2008; 10: 1365-1371.

25 Jung I, Kim SK, Kim M, Han YM, Kim YS, Kim D et al. H2B

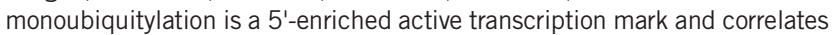
with exon-intron structure in human cells. Genome Res 2012; 22: $1026-1035$

26 Piunti A, Shilatifard A. Epigenetic balance of gene expression by Polycomb and COMPASS families. Science 2016; 352: aad9780.

27 Shilatifard A. The COMPASS family of histone H3K4 methylases: mechanisms of regulation in development and disease pathogenesis. Annu Rev Biochem 2012; 81: 65-95.

28 Kizer KO, Phatnani HP, Shibata Y, Hall H, Greenleaf AL, Strahl BD. A novel domain in Set2 mediates RNA polymerase II interaction and couples histone H3 K36 methylation with transcript elongation. Mol Cell Biol 2005; 25: 3305-3316.

29 Krogan NJ, Kim M, Tong A, Golshani A, Cagney G, Canadien V et al. Methylation of histone $\mathrm{H} 3$ by Set2 in Saccharomyces cerevisiae is linked to transcriptional elongation by RNA polymerase II. Mol Cell Biol 2003; 23 : 4207-4218.

30 Li J, Moazed D, Gygi SP. Association of the histone methyltransferase Set2 with RNA polymerase II plays a role in transcription elongation. $\mathrm{J} \mathrm{Biol} \mathrm{Chem}$ 2002; 277: 49383-49388.

31 Schaft D, Roguev A, Kotovic KM, Shevchenko A, Sarov M, Shevchenko A et al. The histone 3 lysine 36 methyltransferase, SET2, is involved in transcriptional elongation. Nucleic Acids Res 2003; 31: 2475-2482.

32 Radman-Livaja M, Quan TK, Valenzuela L, Armstrong JA, van Welsem T, Kim T et al. A key role for Chd1 in histone $\mathrm{H} 3$ dynamics at the $3^{\prime}$ ends of long genes in yeast. PLoS Genet 2012; 8: e1002811.

33 Lin LJ, Minard LV, Johnston GC, Singer RA, Schultz MC. Asf1 can promote trimethylation of H3 K36 by Set2. Mol Cell Biol 2010; 30: 1116-1129.

34 Du HN, Fingerman IM, Briggs SD. Histone H3 K36 methylation is mediated by a trans-histone methylation pathway involving an interaction between Set2 and histone H4. Genes Dev 2008; 22: 2786-2798.

35 Shi Y, Lan F, Matson C, Mulligan P, Whetstine JR, Cole PA et al. Histone demethylation mediated by the nuclear amine oxidase homolog LSD1. Cell 2004: 119: 941-953.
36 Klose RJ, Yamane K, Bae Y, Zhang D, Erdjument-Bromage H, Tempst P et al. The transcriptional repressor JHDM3A demethylates trimethyl histone H3 lysine 9 and lysine 36. Nature 2006; 442: 312-316.

37 Mosammaparast N, Shi Y. Reversal of histone methylation: biochemical and molecular mechanisms of histone demethylases. Annu Rev Biochem 2010; 79: 155-179.

38 Ramakrishnan S, Pokhrel S, Palani S, Pflueger C, Parnell TJ, Cairns BR et al. Counteracting H3K4 methylation modulators Set1 and Jhd2 co-regulate chromatin dynamics and gene transcription. Nat Commun 2016; 7: 11949.

39 Shi X, Kachirskaia I, Walter KL, Kuo JH, Lake A, Davrazou F et al. Proteome-wide analysis in Saccharomyces cerevisiae identifies several PHD fingers as novel direct and selective binding modules of histone H3 methylated at either lysine 4 or lysine 36. J Biol Chem 2007; 282: 2450-2455.

40 Bian C, Xu C, Ruan J, Lee KK, Burke TL, Tempel W et al. Sgf29 binds histone H3K4me2/3 and is required for SAGA complex recruitment and histone H3 acetylation. EMBO J 2011; 30: 2829-2842.

41 Loewith R, Meijer M, Lees-Miller SP, Riabowol K, Young D. Three yeast proteins related to the human candidate tumor suppressor p33(ING1) are associated with histone acetyltransferase activities. Mol Cell Biol 2000; 20: 3807-3816.

42 Howe L, Kusch T, Muster N, Chaterji R, Yates JR 3rd, Workman JL. Yng1p modulates the activity of Sas $3 p$ as a component of the yeast NuA3 Hhistone acetyltransferase complex. Mol Cell Biol 2002; 22: 5047-5053.

43 Taverna SD, Ilin S, Rogers RS, Tanny JC, Lavender H, Li H et al. Yng1 $\mathrm{PHD}$ finger binding to $\mathrm{H} 3$ trimethylated at $\mathrm{K} 4$ promotes NuA3 HAT activity at K14 of $\mathrm{H} 3$ and transcription at a subset of targeted ORFs. Mol Cell 2006; 24: 785-796.

44 Martin DG, Baetz K, Shi X, Walter KL, MacDonald VE, Wlodarski MJ et al. The Ynglp plant homeodomain finger is a methyl-histone binding module that recognizes lysine 4-methylated histone H3. Mol Cell Biol 2006; 26: 7871-7879.

45 Choy JS, Tobe BT, Huh JH, Kron SJ. Yng2p-dependent NuA4 histone $\mathrm{H} 4$ acetylation activity is required for mitotic and meiotic progression. J Biol Chem 2001; 276: 43653-43662.

46 Vermeulen M, Mulder KW, Denissov S, Pijnappel WW, van Schaik FM, Varier RA et al. Selective anchoring of TFIID to nucleosomes by trimethylation of histone H3 lysine 4. Cell 2007; 131: 58-69.

47 Saksouk N, Avvakumov N, Champagne KS, Hung T, Doyon Y, Cayrou C et al. HBO1 HAT complexes target chromatin throughout gene coding regions via multiple PHD finger interactions with histone $\mathrm{H} 3$ tail. $\mathrm{Mol}$ Cell 2009; 33: 257-265.

48 Hung T, Binda O, Champagne KS, Kuo AJ, Johnson K, Chang HY et al. ING4 mediates crosstalk between histone $\mathrm{H} 3 \mathrm{~K} 4$ trimethylation and H3 acetylation to attenuate cellular transformation. Mol Cell 2009; 33: 248-256.

49 Loewith R, Smith JS, Meijer M, Williams TJ, Bachman N, Boeke JD et al. Pho23 is associated with the Rpd3 histone deacetylase and is required for its normal function in regulation of gene expression and silencing in Saccharomyces cerevisiae. J Biol Chem 2001; 276: 24068-24074.

50 Keogh MC, Kurdistani SK, Morris SA, Ahn SH, Podolny V, Collins SR et al. Cotranscriptional set2 methylation of histone $\mathrm{H} 3$ lysine 36 recruits a repressive Rpd3 complex. Cell 2005; 123: 593-605.

51 Carrozza MJ, Li B, Florens L, Suganuma T, Swanson SK, Lee KK et al. Histone $\mathrm{H} 3$ methylation by Set2 directs deacetylation of coding regions by Rpd3S to suppress spurious intragenic transcription. Cell 2005; 123: 581-592.

52 Shi X, Hong T, Walter KL, Ewalt M, Michishita E, Hung T et al. ING2 PHD domain links histone $\mathrm{H} 3$ lysine 4 methylation to active gene repression. Nature 2006; 442: 96-99.

53 Yang SY, Baxter EM, Van Doren M. Phf7 controls male sex determination in the Drosophila germline. Dev Cell 2012; 22: 1041-1051.

$54 \mathrm{Li}$ J, Chu M, Wang S, Chan D, Qi S, Wu M et al. Identification and characterization of nardilysin as a novel dimethyl H3K4-binding protein involved in transcriptional regulation. J Biol Chem 2012; 287: 10089-10098.

55 Pijnappel WW, Schaft D, Roguev A, Shevchenko A, Tekotte H, Wilm M et al. The S. cerevisiae SET3 complex includes two histone deacetylases, Hos2 and Hst1, and is a meiotic-specific repressor of the sporulation gene program. Genes Dev 2001; 15: 2991-3004.

56 Gilbert TM, McDaniel SL, Byrum SD, Cades JA, Dancy BC, Wade $\mathrm{H}$ et al. A PWWP domain-containing protein targets the NuA3 acetyltransferase 
complex via histone $\mathrm{H} 3$ lysine 36 trimethylation to coordinate transcriptional elongation at coding regions. Mol Cell Proteomics 2014; 13: 2883-2895.

57 Smolle M, Venkatesh S, Gogol MM, Li H, Zhang Y, Florens L et al. Chromatin remodelers Isw1 and Chd 1 maintain chromatin structure during transcription by preventing histone exchange. Nat Struct Mol Biol 2012; 19: 884-892.

58 Joshi AA, Struhl K. Eaf3 chromodomain interaction with methylated H3-K36 links histone deacetylation to Pol II elongation. Mol Cell 2005; 20 : 971-978.

59 Lan F, Collins RE, De Cegli R, Alpatov R, Horton JR, Shi X et al. Recognition of unmethylated histone $\mathrm{H3}$ lysine 4 links BHC80 to LSD1mediated gene repression. Nature 2007; 448: 718-722.

60 Venkatesh S, Smolle M, Li H, Gogol MM, Saint M, Kumar S et al. Set2 methylation of histone $\mathrm{H} 3$ lysine 36 suppresses histone exchange on transcribed genes. Nature 2012; 489: 452-455.

61 Wang A, Kurdistani SK, Grunstein M. Requirement of Hos2 histone deacetylase for gene activity in yeast. Science 2002; 298: 1412-1414.

62 Kim T, Xu Z, Clauder-Munster S, Steinmetz LM, Buratowski S. Set3 HDAC mediates effects of overlapping noncoding transcription on gene induction kinetics. Cell 2012; 150: 1158-1169.

63 Keogh MC, Podolny V, Buratowski S. Bur1 kinase is required for efficient transcription elongation by RNA polymerase II. Mol Cell Biol 2003; 23: 7005-7018.

64 Biswas D, Dutta-Biswas R, Mitra D, Shibata Y, Strahl BD, Formosa T et al. Opposing roles for Set2 and yFACT in regulating TBP binding at promoters. EMBO J 2006; 25: 4479-4489.

65 Quan TK, Hartzog GA. Histone H3K4 and K36 methylation, Chd1 and Rpd3S oppose the functions of Saccharomyces cerevisiae Spt4-Spt5 in transcription. Genetics 2010; 184: 321-334.

66 Drouin S, Laramee L, Jacques PE, Forest A, Bergeron M, Robert F. DSIF and RNA polymerase II CTD phosphorylation coordinate the recruitment of Rpd3S to actively transcribed genes. PLoS Genet 2010; 6: e1001173.

67 Govind CK, Qiu H, Ginsburg DS, Ruan C, Hofmeyer K, Hu C et al. Phosphorylated Pol II CTD recruits multiple HDACs, including Rpd3C(S), for methylation-dependent deacetylation of ORF nucleosomes. $\mathrm{Mol} \mathrm{Cell}$ 2010; 39: 234-246.

68 Li B, Gogol M, Carey M, Lee D, Seidel C, Workman JL. Combined action of PHD and chromo domains directs the Rpd3S HDAC to transcribed chromatin. Science 2007; 316: 1050-1054.

69 McDaniel SL, Fligor JE, Ruan C, Cui H, Bridgers JB, DiFiore JV et al. Combinatorial histone readout by the dual plant homeodomain (PHD) fingers of Rcol mediates Rpd3S chromatin recruitment and the maintenance of transcriptional fidelity. J Biol Chem 2016; 291: 14796-14802.
70 Ruan C, Cui H, Lee CH, Li S, Li B, Homodimeric PHD. Domain-containing Rcol subunit constitutes a critical interaction hub within the Rpd3S histone deacetylase complex. J Biol Chem 2016; 291: 5428-5438.

71 Cheung V, Chua G, Batada NN, Landry CR, Michnick SW, Hughes TR et al. Chromatin- and transcription-related factors repress transcription from within coding regions throughout the Saccharomyces cerevisiae genome. PLoS Biol 2008; 6: e277.

72 Li B, Gogol M, Carey M, Pattenden SG, Seidel C, Workman JL. Infrequently transcribed long genes depend on the Set2/Rpd3S pathway for accurate transcription. Genes Dev 2007; 21: 1422-1430.

73 Venkatesh S, Li H, Gogol MM, Workman JL. Selective suppression of antisense transcription by Set2-mediated H3K36 methylation. Nat Commun 2016; 7: 13610.

74 Kim JH, Lee BB, Oh YM, Zhu C, Steinmetz LM, Lee Y et al. Modulation of mRNA and IncRNA expression dynamics by the Set2-Rpd3S pathway. Nat Commun 2016; 7: 13534.

75 Tan-Wong SM, Zaugg JB, Camblong J, Xu Z, Zhang DW, Mischo HE et al. Gene loops enhance transcriptional directionality. Science 2012; 338: 671-675.

76 Bilodeau S, Kagey MH, Frampton GM, Rahl PB, Young RA. SetDB1 contributes to repression of genes encoding developmental regulators and maintenance of ES cell state. Genes Dev 2009; 23: 2484-2489.

77 Shi Y. Histone lysine demethylases: emerging roles in development, physiology and disease. Nat Rev Genet 2007; 8: 829-833.

78 Greer EL, Shi Y. Histone methylation: a dynamic mark in health, disease and inheritance. Nat Rev Genet 2012; 13: 343-357.

79 Varier RA, Timmers HT. Histone lysine methylation and demethylation pathways in cancer. Biochim Biophys Acta 2011; 1815: 75-89.

80 Kutateladze TG. ING family of tumor suppressors. Curr Drug Targets 2009; 10: 384 .

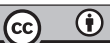

This work is licensed under a Creative Commons Attribution 4.0 International License. The images or other third party material in this article are included in the article's Creative Commons license, unless indicated otherwise in the credit line; if the material is not included under the Creative Commons license, users will need to obtain permission from the license holder to reproduce the material. To view a copy of this license, visit http://creativecommons. org/licenses/by/4.0/ 